\title{
Study on Flexible Polishing Force and Polishing Temperature of Flap Disc
}

De Liu ( $\nabla$ ldhnbc@163.com )

Wenzhou University

Xiaoming Pan

Wenzhou University

Zhiyang Gu

Wenzhou Polytechnic

Hui Qiu

Wenzhou University

\section{Research Article}

Keywords: Flexible polishing, Abrasive cutting, Polishing force, Polishing heat, Specific polishing energy

Posted Date: July 12th, 2021

DOI: https://doi.org/10.21203/rs.3.rs-626617/v1

License: @ (i) This work is licensed under a Creative Commons Attribution 4.0 International License. Read Full License 


\title{
Study on flexible polishing force and polishing temperature of flap disc
}

\author{
De Liu ${ }^{1}$, Xiaoming Pan ${ }^{*}$, Zhiyang $\mathrm{Gu}^{2}$ and Hui Qiu ${ }^{1}$ \\ ${ }^{1}$ College of Mechanical and Electrical Engineering, Wenzhou University, Wenzhou 325035, China \\ ${ }^{2}$ Department of electrical and Electronic Engineering, Wenzhou Polytechnic, Wenzhou 325035, China
}

\begin{abstract}
Polishing determines the final surface quality of the aero engine, which have great influence on its working performance and working life. By analyzing the structure and working principle of the flexible self-adaptive polishing platform of the blisk, the abrasive cutting model of the flap disc is established. The theoretical calculation of the effect of elastic deformation during the polishing process on the contact length of flap disc and blisk. The model of polishing force, polishing heat and temperature field during the polishing process of the flap disc are established and analyzed. Single factor method is used to analyze the influence of process parameters on polishing force, polishing temperature, roughness and specific polishing energy. Finally, the polishing test shows that the optimized process parameters improve the polished surface quality and meet the requirements of the blade polishing process.
\end{abstract}

Keywords: Flexible polishing; Abrasive cutting; Polishing force; Polishing heat; Specific polishing energy

\section{Introduction}

The Integral blisk of aero engine has the characteristics of complex blade surface, thin blade body, long blade extension, poor rigidity and uneven processing allowance, etc. Therefore, the polishing process of the blisk is demanding. There are still many deficiencies in the polishing equipment and process technology of the complex curved surface. Manual polishing is still the main processing method. The processing efficiency is low, the working environment is hard, the workpiece processing accuracy and surface quality are greatly affected by the worker's experience. The processing accuracy is low and the processing surface consistency is difficult to guarantee. Therefore, the polishing process has attracted attention and a series of studies have been done.

Wu studied the polishing of M300 Mold steel with elastic abrasive, and the polishing parameters was optimized by Taguchi method so that educe the surface roughness [1]. Xian studied the vibration characteristics of the polishing blade with the abrasive cloth wheel, and the blade damage caused by vibration was reduced [2]. In order to control the vibration of polishing process, Lin proposed a method of predicting the value of polishing vibration signal. The empirical model of process parameters for polishing vibration was established [3]. Aiming at developing a mobile robot system to perform rust removal,

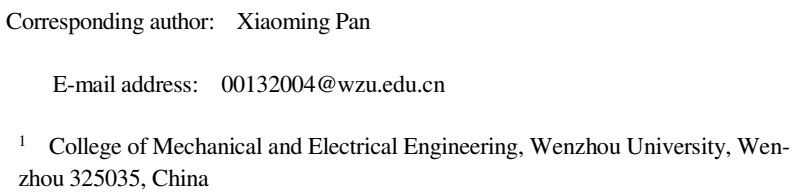

Wang studied the spiral brushing force through theory and finite element method [4]. Liu studied the surface roughness and residual stress of the polished aero engine blisk and blade surfaces, optimized the polishing process, and improved the surface integrity [5-7]. Zhang studied the polishing of blisk with the abrasive belt, optimized the process parameters, and the surface roughness was reduced [8]. Liu optimized the tool path and the shape of flap disc to avoid the occurrence of knife marks [9]. Wang used the abrasive cloth wheel to polish the free-form surface. By planning the direction of the knife axis, the polishing surface roughness was reduced [10]. Huai established the prediction model for the polishing surface roughness of abrasive cloth wheel, and the process parameters were optimized to reduce the polishing surface roughness $[11,12]$. Ma polished the additive titanium alloy by means of laser polishing, and the laser polished surface and cross-section subsurface were analyzed by white light interference, confocal microscope, focused ion beam, scanning electron microscope, energy dispersive spectrometer and X-ray diffraction [13]. Xiao illustrated the methodology of CABP and established an equation for microdisplacement in constant-load adaptive control to improve the accuracy and the consistency of the polishing blade surface quality [14]. Tian studied the automatic polishing of surfaces by robots, analyzed the relationship between polishing pressure and removal efficiency, and the quality of polished surfaces were improved by controlling the pressure [15]. Janus used two polishing methods to process different nanomaterials, compared the effects of surface roughness and micro-morphology before and after polishing [16]. Antonson and gönülol compared the effects of different polishing systems on composite materials, compared the effects of surface roughness and gloss, etc. The best polishing method was determined $[17,18]$. Evgueni reviewed the progress of laser polishing technology in 
theoretical and experimental research, and emphasized the processing performance defined by the surface roughness before and after polishing [19]. Berger investigated the influence of filler size and finishing systems on the surface roughness [20]. Zhang established the prediction model of roughness for abrasive belt polishing, optimized the process parameters and improved the quality of the polished blisk [21].

Northwestern Polytechnical University developed a five-axis CNC polishing machine independently, which has both the trajectory precision of the $\mathrm{CNC}$ machine tool and the control unit of the polishing force. The flap disc with simple structure and good elasticity is used as an abrasive tool. The flap disc can be deepened into the blisk of the aero engine for adaptive polishing, which can effectively reduce the occurrence of over-polishing and under-polishing[22].

\section{Working principle of polishing machine}

A five-axis CNC polishing machine with a gantry structure is used as the polishing equipment. The experimental platform is a polishing system that integrates polishing force control and micro-displacement adjustment. The integral blisk polishing machine is shown in Fig. 1. The machine tool contains five coordinates. The grinding spindle can complete linear motion in the vertical plane composed of $\mathrm{X}$-axis and $\mathrm{Z}$-axis, swing motion around A-axis within a certain range of angle. The rotating worktable carries the blisk, rotates around the coordinate $\mathrm{C}$-axis on the same horizontal plane, and moves linearly along the $\mathrm{Y}$ axis. Two rotary coordinate axes $\mathrm{A}$ and $\mathrm{C}$, three linear coordinate axes $\mathrm{X}, \mathrm{Y}, \mathrm{Z}$ cooperate to complete the linear movement and rotary movement of the workpiece and the grinding spindle, so that control the processing track of the workpiece. The main components of the polishing experiment platform are the column that supports the workpiece, the box for loading the spindle, the spindle for polishing the workpiece, the rotatable work platform, the base, the machine tool guard, the clamping tool, the shaft guard, and the servo motor that controls the movement, etc. The electro-spindle and its flexible control mechanism are located in the spindle box. The electro-spindle mechanism is

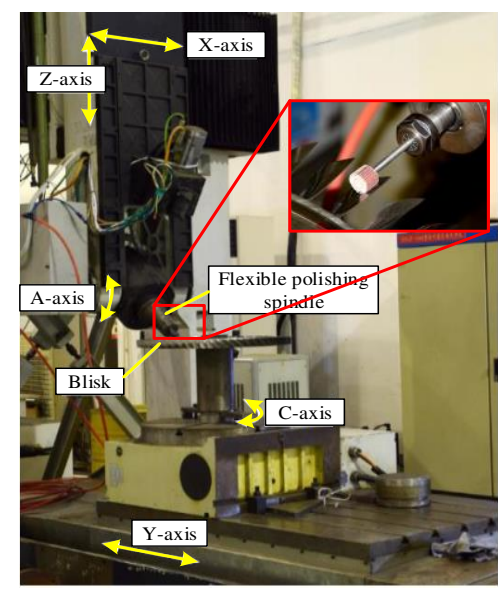

(a) Polishing machine controlled by the servo motor that controls the movement of the A-axis to complete the swing movement, and the range of swing angle is $0 \sim 90^{\circ}$. The blisk is fixed on the worktable by fixture, and the $\mathrm{C}$-axis servo motor controls its rotation, and the range of rotation angle is $0 \sim 360^{\circ}$.

In order to achieve multi-axis linkage and control the movement trajectory accurately, the integral blisk polishing experiment platform adopts siemens system, simodrive $611 \mathrm{D}$ power module, simatic S7-200 logic controller (PLC) to form a fully digital control system. The computer control is used as the numerical control system, which has the advantages of modularization and digital control. It is convenient to complete information interaction with other equipment, so as to efficiently control the equipment to complete the processing of the blisk.

The surface of the blisk is a free-form surface. It is difficult to accurately plan the polishing trajectory, which leads to deviations between the theoretical polishing path and the actual polishing path. For this phenomenon, the machine tool spindle can monitor the machining process in real time, and the spindle mechanism can perform floating adjustment of the polishing process, so that the micro-displacement between the spindle mechanism and the tool can be automatically adjusted, and the elastic contact between the tool and the workpiece can be ensured by the floating adjustment of the polishing force. Besides, the spindle mechanism can set the difference interval between the actual polishing force and the theoretical polishing force according to the processing requirements to ensure the dynamic and stable control of the polishing force and reduce the processing error caused by the unreasonable polishing path.

The main components of elastic grinding spindle to realize flexible polishing include control cylinder and displacement sensor to realize displacement adjustment, spindle outer sleeve and inner sleeve for protecting and supporting components, swing support shaft, cylinder support cover, spring chuck for positioning, electric spindle that provides power for tool rotation. The structure of the flexible grinding spindle is shown in Fig. 2. The main shaft is installed with a sleeve embedded structure, and the control cylinder runs through the inner sleeve of the main shaft and is located between the inner sleeve of the

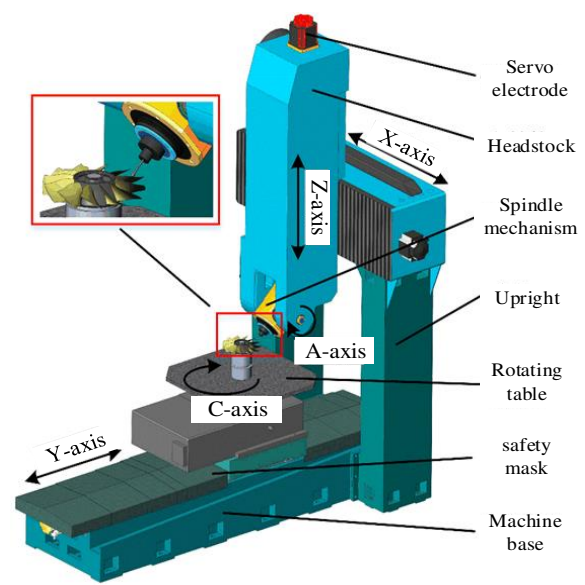

(b) Three-dimensional model

Fig. 1 The integral blisk polishing machine 
main shaft and the central main shaft. The cylinder and the cylinder support cover connect the spindle inner sleeve and the spindle outer sleeve. Three groups of cylinders are evenly distributed along the radial direction of the polishing spindle at a certain angle. There is a cylinder at the top of the spindle along the axial direction. The four groups of cylinders control the grinding spindle to achieve radial micro-movement. The cylinders cooperate with each other to complete the adjustment of the spindle's pose. The displacement sensor and the cylinder are arranged in pairs, and they are installed on both sides of the main shaft in parallel to detect and adjust the displacement of the main shaft. The swing support shaft of the main shaft is located in the main shaft box to realize the swing of the main shaft along the A-axis.

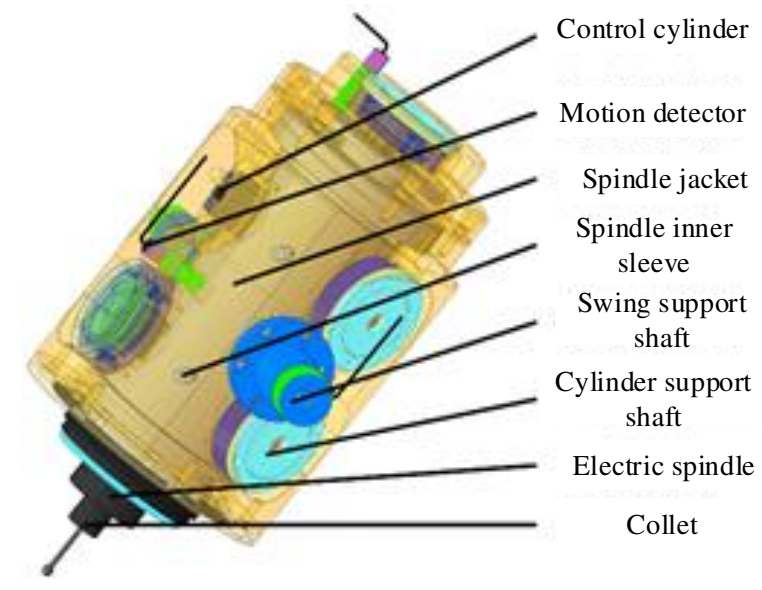

Fig. 2 Structure distribution of flexible grinding spindle

The grinding spindle is a key processing unit for the machine tool to realize flexible polishing. It can realize elastic contact between the tool and the workpiece, reducing the occurrence of over-polishing or under-polishing. The displacement sensor on the grinding spindle monitors the changes of spindle pose in time, collects relevant data signals and inputs them into the industrial control system. The data signal is analyzed through the control algorithm, and the output signal command is used to control the expansion and contraction of the cylinder to realize the micro-displacement adjustment of the spindle. Through the adjustment of the spindle's pose, the stable control of the spindle polishing force is ensured. The micro-displacement adjustment can effectively compensate the deviation between the actual polishing path and the theoretical polishing path caused by tool wear and inconsistent machining allowance.

\section{Flexible polishing mechanism of flap disc}

\subsection{Technical characteristics of flap disc polishing}

The experiment chooses the flap disc as the polishing tool. In the process of polishing the blisk, the main characteristics of the flap disc are as follows.

(1) The flap disc is a flexible polishing method. Firstly, the flap disc abrasive binder has a certain elasticity. In addition, the flap disc selects a cloth base with good flexibility as the abrasive carrier. During the polishing process, the rotating main shaft drives the flap disc to rotate at high speed, and the abrasive cloth sheet is fully stretched under the action of centrifugal force, so that the flap disc and the workpiece are fully attached to achieve shape-followed polishing. This polishing method has a larger contact area and a more uniform polishing force, which can effectively reduce the occurrence of over-polishing or under-polishing, improve the surface quality of polished freeform surfaces.

(2) The flap disc and the workpiece are in elastic contact, and system has good stability during polishing. The polishing force of the flap disc is relatively small, so that the deformation of the workpiece is relatively small, and the workpiece with poor rigidity can also have a good polishing effect. At the same time, the flap disc rotates at a high speed, the removed abrasive particles are separated from the abrasive tool under the action of centrifugal force, which is helpful to reduce the polishing temperature and prevents burns on the workpiece.

(3) The flap disc has a simple structure, does not require a complicated external support structure. In addition, due to the small size of the flap disc, it can go deep into the flow passage of the blisk, so that achieve a good polishing effect on the freeform surface.

\subsection{Abrasive cutting process model}

The flap disc is a kind of micro-edged cutting tool. In the process of material removal, the tool rotates at high speed, so that a large number of fine abrasive particles are drawn across the surface of the workpiece to complete the removal of the material. The removal effect of a large number of abrasive particles are superimposed to complete the polishing process. In order to understand the polishing mechanism of the flap disc, it is necessary to analyze the cutting process of a single abrasive particle.

The material removal process model of a single abrasive particle of the flap disc is shown in Fig. 3. According to the different material removal effect of abrasive particles, it can be divided into sliding, ploughing and cutting.

During the polishing process, the abrasive particles and the surface of the workpiece are squeezed to generate heat and deformation, which is the main cause of thermal stress and deformation stress. When the contact force between the abrasive particles and the material is small, and the deformation is recoverable elastic deformation, it is in the sliding stage. When the abrasive particles squeeze the material and the material produces irreversible plastic deformation without material falling off, it is in the plough stage. If the abrasive particles cut the workpiece, the removed material fragments are separated from the workpiece and flow out along the rake face of the abrasive particle micro-edge, it is in the cutting stage. According to the cutting performance of the material and the sharpness of the abrasive micro-edge, it will affect the processing stage of the material removal model.

The rotation speed of the flap disc also affects the formation of friction, ploughing and cutting of abrasive particles on the cutting surface. The higher the rotating speed, the smaller the 

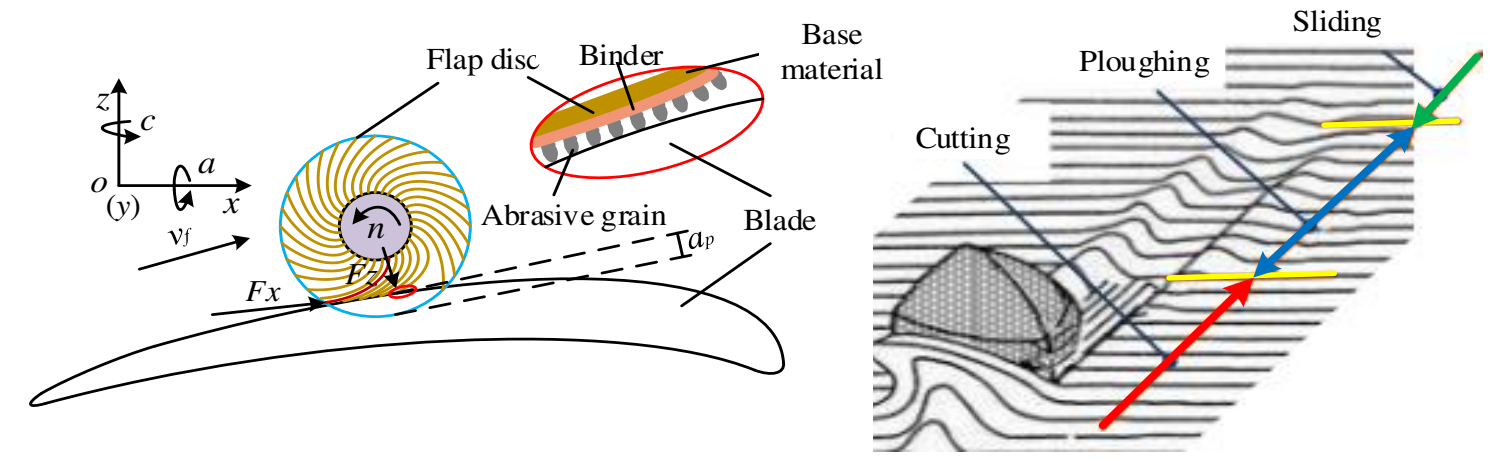

Fig. 3 Polishing abrasive grain cutting model of flap disc

elastic-plastic zone. The actual cutting amount of a single abrasive partical is different, and the elastoplastic deformation zone is also different. Because the binder of the abrasive particles has a certain elasticity, the abrasive particles will retreat under the action of cutting force, which makes the actual contact curve of the abrasive particles and the workpiece different from the theoretical contact curve. In addition, in the process of material removal, the force between the abrasive particles and the workpiece causes deformation. When the abrasive particles are separated from the material, the deformation of the material is restored, so that the theoretical cutting path does not match the actual profile, which affects the accuracy and surface roughness. The elastic retreat and elastoplastic contact area of abrasive particles are shown in Fig. 4.

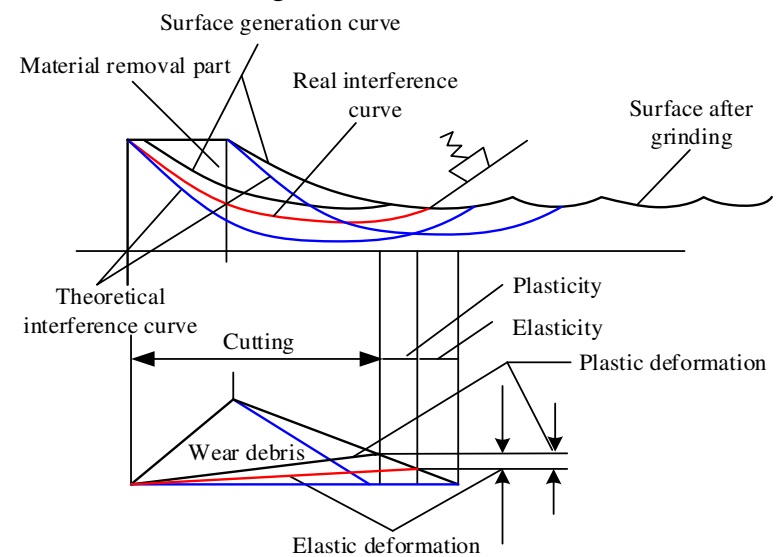

Fig. 4 The elastic retreat and elastoplastic contact area of abrasive particles

\subsection{The influence of elastic deformation on the contact length}

In the process of polishing the blade, the flap disc has good flexibility, which can be regarded as an elastic body. Under the action of the normal polishing force, it will cause the flap disc and the workpiece to produce contact displacement $\delta_{e}$, the abrasive grains and the workpiece to produce contact displacement $\delta_{e}$ ", the sum of the two $\delta_{e}$ is shown in Eq. (1).

$\delta_{e}=\delta_{e}^{\prime}+\delta_{e}^{\prime \prime}$

The contact displacement of the flap disc, abrasive particles and the workpiece can be solved by the elastic contact model. The normal polishing force causes the displacement of the flap disc and the workpiece $\delta_{e}$, which can be analyzed according to the contact model that does not consider the cutting depth, Fig. 5(a). The contact displacement between abrasive particles and workpiece $\delta_{e}$ ", which can be analyzed according to the contact model that consider the cutting depth, as in Fig. 5(b). Where $d_{s}$ is the diameter of the flap disc.

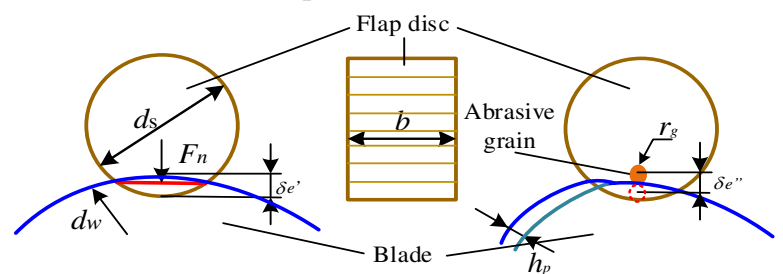

(a) Contact displacement $\delta e^{\text {, }}$

(b) Contact displacement $\delta e$ "

Fig. 5 The grinding contact model

According to the contact model that does not consider the cutting depth, the contact displacement between the flap disc and the workpiece is calculated as shown in Eq. (2). Where $F_{n}$ is normal cutting force, $b$ is the width of flap disc, $v_{w}$ and $v_{s}$ are the poisson's ratio of the workpiece and the flap disc. $E_{w}$ and $E_{s}$ are the elastic modulus of workpiece and the flap disc.

$\delta_{e}^{\prime}=\frac{2 F_{n}}{\pi b}\left[\frac{1}{3}\left(\frac{1-v_{w}^{2}}{E_{w}}+\frac{1-v_{s}^{2}}{E_{s}}\right)+\frac{1-v_{w}^{2}}{E_{w}} \ln \frac{4 d_{w}}{l}+\frac{1-v_{s}^{2}}{E_{s}} \ln \frac{4 d_{s}}{l}\right]$

The abrasive grain cutting edge is in contact with the workpiece and produces a contact displacement. The abrasive grain is regarded as an elastic sphere with a radius of $r_{g}$. By considering the contact model of cutting depth and the abrasive grain cutting edge, the force generated by the abrasive grain displacement $\delta_{e}$ ", is solved, as shown in Eq. (3).

$\delta_{e}^{\prime \prime}=\left[\frac{9}{16}\left(\frac{1-v_{w}^{2}}{E_{w}}+\frac{1-v_{s}^{2}}{E_{s}}\right) F_{g}\right]^{1 / 3}$

The contact arc length between the flap disc and the workpiece is $l_{s}$, as shown in Eq. (4). where "+" means polishing the outer arc at the back of the blade, and "-" means polishing the inner arc at the basin of the blade, $h_{p}$ is the cutting depth of abrasive grains, $d_{w}$ and $d_{s}$ are the contact diameterb)f workpiece and flap disc. 
$l_{s}=\sqrt{\frac{d_{w} d_{s}}{d_{w} \pm d_{s}}}\left(\sqrt{h_{p}+\delta_{e}}+\sqrt{\delta_{e}}\right)$

\section{Study on polishing force and polishing temperature}

\subsection{Analytical model of polishing force}

Polishing force is an important characterizing parameter of the polishing process, which has an important influence on the surface quality and machining accuracy. In order to establish the analytical model of polishing force, it is necessary to determine the contact dynamic effective grinding edge number of the contact surface between the flap disc and workpiece in unit area and the average cutting area within the contact arc length.

The dynamic effective number of grinding edges on the interface between flap disc and workpiece $N_{d}$ is shown in Eq. (5), the contact dynamic effective grinding edge number of the contact surface between the flap disc and workpiece in unit length $N_{d}(l)$ is shown in Eq. (6), where $A_{n}$ is the proportional coefficient related to the number of static grinding edges, $C_{e}$ is the coefficient related to abrasive density, $\alpha$ and $\beta$ are constant terms.

$$
\begin{aligned}
& N_{d}=A_{n}\left(c_{e}\right)^{\beta}\left(\frac{v_{w}}{v_{s}}\right)^{\alpha}\left(\frac{h_{p}}{d_{s}}\right)^{\frac{\alpha}{2}} \\
& N_{d}(l)=N_{d}\left(\frac{l}{l_{s}}\right)^{\alpha}=A_{n}\left(c_{e}\right)^{\beta}\left(\frac{v_{w}}{v_{s}}\right)^{\alpha}\left(\frac{h_{p}}{d_{s}}\right)^{\frac{\alpha}{2}}\left(\frac{l}{l_{s}}\right)^{\alpha}
\end{aligned}
$$

The average value of the maximum material removal crosssectional area $\bar{A}_{\text {max }}$ is shown in Eq. (7). The average material removal area $\bar{A}(l)$ at contact arc length $l$ is shown in Eq. (8).

$\bar{A}_{\text {max }}=\frac{2}{A_{n}}\left(c_{e}\right)^{-\beta}\left(\frac{v_{w}}{v_{s}}\right)^{1-\alpha}\left(\frac{h_{p}}{d_{s}}\right)^{\frac{1-\alpha}{2}}$

$\bar{A}(l)=\bar{A}_{\max }\left(\frac{l}{l_{s}}\right)^{1-\alpha}=\frac{2}{A_{n}}\left(c_{e}\right)^{-\beta}\left(\frac{v_{w}}{v_{s}}\right)^{1-\alpha}\left(\frac{h_{p}}{d_{s}}\right)^{\frac{1-\alpha}{2}}\left(\frac{l}{l_{s}}\right)^{1-\alpha}$

The radial polishing force between the flap disc and the workpiece is shown in Eq. (9), where $K$ and $n$ are proportional coefficients related to abrasive particles.

$F_{n}^{\prime}=K[\bar{A}(l)]^{n} N_{d}(l)$

Integrate the entire arc length to get the radial polishing force $F_{n}$, as is in Eq. (10).

$F_{n}(l)=K \int_{0}^{L_{S}}[\bar{A}(l)]^{n} N_{d}(l) d l$

Substituting Eq. (6) and Eq. (8) into Eq. (10), Eq. (11) can be obtained. Where $\varepsilon=\frac{1}{2}[(1+n)+\alpha(1-n)], \quad \gamma=\beta(1-$ $n)$ 。

$$
F_{n}(l)=K\left(c_{e}\right)^{\gamma}\left(\frac{v_{w}}{v_{s}}\right)^{2 \epsilon-1}\left(h_{p}\right)^{\varepsilon}\left(d_{s}\right)^{1-\varepsilon}
$$

Polishing force is mainly composed of cutting force and friction force. Based on the mathematical model of grinding and polishing force, the polishing force model is expressed as two parts, as in Eq. 12. Where $F_{t}$ is tangential force, $F_{n c}$ and $F_{t c}$ are the radial force and tangential force caused by cutting deformation, $F_{n s}$ and $F_{t s}$ are the radial force and tangential force caused by friction, $\bar{\delta}$ is the actual contact area between the abrasive particles and the workpiece, $\bar{P}$ is the average contact pressure between abrasive particles and workpiece, $\varphi=$ $\frac{\pi}{4 \tan \theta}$
$\left\{\begin{array}{c}F_{n}=F_{n c}+F_{n s}=K \sum A l_{s}+\underset{(4)}{N} \bar{\delta} \bar{P} \\ F_{t}=F_{t c}+F_{t s}=\varphi K \sum A l_{s}+N \mu \bar{\delta} \bar{P}\end{array}\right.$

Material cutting area per unit area of abrasive particles is shown in Eq. (13). The average polishing pressure of the abrasive particles is shown in Eq. (14), and

$\sum A l_{s}=\frac{v_{w}}{v_{s}} h_{p}$

$\bar{P}=\frac{d F_{n}}{d \bar{\delta}}$

The total number of abrasive grains per unit polishing area of the flap disc is $N$, as in Eq. (15).

$N=\int_{0}^{l_{s}} N_{d} d l=\frac{A}{1+\alpha}\left(C_{e}\right)^{\gamma}\left(\frac{v_{w}}{v_{s}}\right)^{\alpha}\left(h_{p}\right)^{\frac{1+\alpha}{2}}\left(d_{s}\right)^{\frac{1-\alpha}{2}}$

Substituting Eq. (13) and Eq. (15) into Eq. (12), the radial and tangential polishing forces per unit area can be obtained, as in Eq. (16).

$$
\left\{\begin{array}{c}
F_{n}=K\left(\frac{v_{w}}{v_{s}} h_{p}\right)+\frac{A_{n} \bar{\delta} \bar{P}}{1+\alpha}\left(c_{e}\right)^{\beta}\left(\frac{v_{w}}{v_{s}}\right)^{\alpha}\left(h_{p} \frac{1+\alpha}{2} d_{s}^{\frac{1-\alpha}{2}}\right) \\
F_{t}=\frac{\pi K}{4 \tan \theta}\left(\frac{v_{w}}{v_{s}} h_{p}\right)+\frac{\mu A_{n} \bar{\delta} \bar{P}}{1+\alpha}\left(c_{e}\right)^{\beta}\left(\frac{v_{w}}{v_{s}}\right)^{\alpha}\left(h_{p}^{\frac{1+\alpha}{2}} d_{s}^{\frac{1-\alpha}{2}}\right)
\end{array}\right.
$$

\subsection{Analytical model of polishing temperature}

The blisk and blades of the aero-enging are made of titanium alloy materials with low thermal conductivity. The polishing heat increases thegfemperature in the polishing area of the workpiece, which has a great influence on the surface quality of the workpiece and the cutting performance of abrasive particles.

(1) During the polishing process, the contact area $A B C D$ of the flap disc and the workpiece moves relative to each other to generate polishing heat, Assuming that this area is a uniform and constant surface heat source, the heat per unit area per unit (8) time is q. $^{\text {tim }}$

(2) During the polishing process of the flap disc, the heat transferred to the blade is $\eta \%$, and the blade is regarded as a semi-infinite heat conductor, and the heat generated by the heat source is $q_{m}=q . \eta \%$.

(3) Generally, the radius of the blade is much larger than the radius of the flap disc, and the contact area $A B C D$ of the flap disc and the blade is regarded as a plane.

(4) The length of surfiod heat source $l(A B)=$ $\sqrt{r_{s}^{2}-\left(r_{s}-a_{p}\right)^{2}}=\sqrt{2 a_{p} r_{s}-a_{p}^{2}}$, where $r_{s}$ is the working radius of flap disc, $a_{p}$ is the amount of compression.

(5) Ignore the influence of material removal depth and tool wear on heat generation.)

According to the analysis of the above polishing process, the heat transfer model of the flap disc polishing blade can be regard as an infinite width and finite length moving surface heat source problem, which can be simplified to a two-dimensional heat conduction problem, as in Fig. 6. 


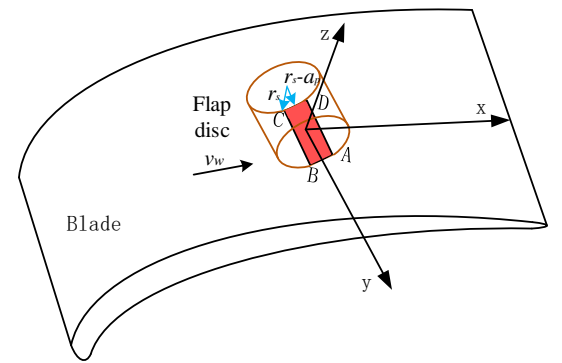

(a)Heat transfer model

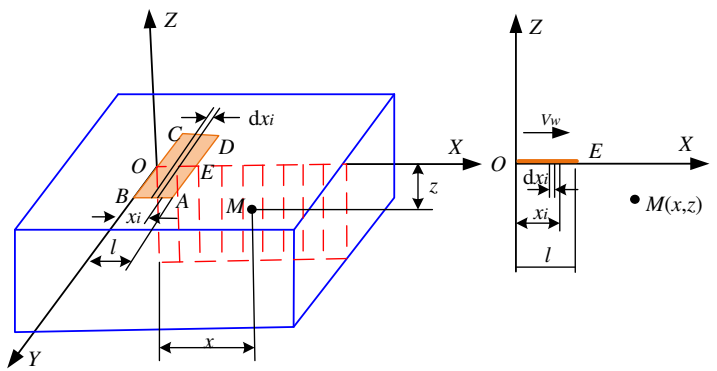

(b) Simplified heat transfer model

Fig. 6 Heat transfer model of blade polishing

Combine Fourier's law of heat conduction with Talau series to analyze the change in temperature $d \theta$ of the microelement body over time $d t$, as is shown in Eq. (17).

$\mathrm{d} \theta=\frac{\Delta Q}{c \rho A d x}=\frac{\lambda}{c \rho} \frac{\partial^{2} \theta(x, t)}{\partial x^{2}} d \mathrm{t}$

A point heat source in an infinite conductor emits instantaneous heat $Q$ at time $t$, and the temperature $\theta$ of an arbitrary point $M(\mathrm{x}, 0, \mathrm{z})$ in the vertical plane of the heat conductor is shown in Eq. (18).

$\theta=\frac{Q}{c \rho(4 \pi a t)} e^{-\frac{r^{2}}{4 a t}}$

At the moment $\mathrm{d} \tau_{i}$, the heat generated by polishing $q_{s} \mathrm{~d} \tau_{i}$ causes an rise in temperature at point $M$, which is regarded as an instantaneous line heat source for calculation.

$\mathrm{d} \theta=\frac{q_{s} \mathrm{~d} \tau_{i}}{c \rho(4 \pi a \tau)} e^{-\frac{\left(x-v \tau_{i}\right)^{2}+z^{2}}{4 a \tau}}$

At the moment of $\mathrm{d} \tau_{i}$, the heating of the sports heat source is regarded as instantaneous heating. The starting time of the action is $\tau_{i}$, and the time from this moment to the observation time $t$ is $\tau$. The distance between the observation point $M$ and the heat source in the $x$ direction is $x-v \tau_{i}$. In order to make the solution of the motion temperature field more convenient, a motion coordinate system is established, $\tau=t-\tau_{i} . x-v \tau_{i}=$ $x-v t+v \tau=X+v \tau$. And Eq. (20) can be obtained.

$\mathrm{d} \theta=\frac{q_{s} \mathrm{~d} \tau_{i}}{c \rho(4 \pi a \tau)} e^{-\frac{(X+v \tau)^{2}+z^{2}}{4 a \tau}}$

During the whole process from $\square \tau_{I}=0$ to $\tau_{I}=t$, the heat source of the moving line causes the temperature rise of point $M$ as in Eq. (21). $\theta=\frac{q_{s}}{4 \pi \lambda} \int_{0}^{t} \frac{d \tau_{i}}{\tau} e^{-\frac{(X+v \tau)^{2}+z^{2}}{4 a \tau}}$

According to the analysis of the two-dimensional heat conduction problem, the surface heat source $\mathrm{ABCD}$ can be regarded as a synthesis of countless linear heat sources, and these linear heat sources move along the $X$ direction at a speed of $v_{w}$. Take the surface heat source $\mathrm{d} X_{i}$ for analysis, as in Fig. 6(b). The surface heat source intensity is $q_{m} \mathrm{~d} X$ (cal/cm.s), the temperature distribution equation is $\theta(x, t), A$ is the cross-sectional area of heat conduction, $\Delta Q$ is the amount of heat change, $\lambda$ and $a$ are constants.

According to the Eq. (21) and the principle of the adiabatic surface caused by the mirror image heat source, the temperature rise of point $M$ in the $x z$ plane of the workpiece affected by the $\mathrm{d} X_{I}$ motion heat source is shown in Eq. (22). Where $K_{0}=\ln \left(v \sqrt{X^{2}+z^{2}} / 4 a\right)-\gamma, \gamma$ is Euler's constant.

$\theta=\frac{q_{m}}{\pi \lambda} \int_{0}^{l} \frac{\left(X-X_{i}\right)}{2 a} K_{0}\left[\frac{v_{w}}{2 a} \sqrt{\left(X-X_{i}\right)^{2}+z^{2}}\right] d X_{i}$

\section{Polishing test and analysis}

\subsection{Experimental conditions}

The five-axis CNC machine is used as the processing equipment, the TC-17 titanium( 1 Tlby blade is the test piece, and the material composition is shown in Table 1. The flap disc is the abrasive tool for polishing test. The dynamic piezoelectric force gauge is fixed on the rotary table through the fixture to measure the polishing force (as in Fig. 7(a)), the

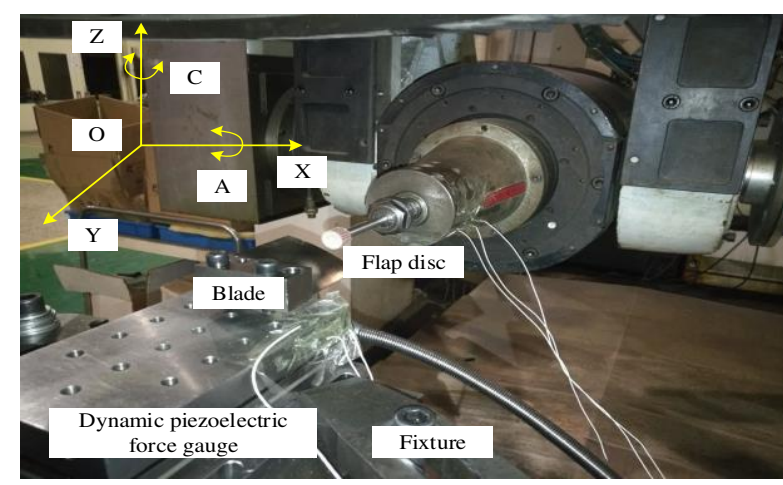

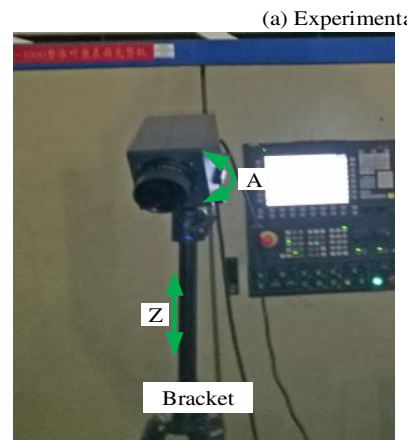

(b) Infrared thermograph

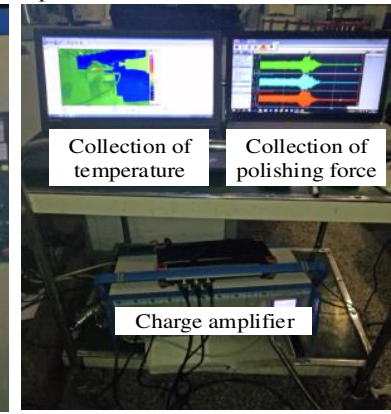

(c) Signal acquisition system
Fig. 7 Measurement of polishing force and temperature 
infrared thermal imager is used to measure the polishing temperature (as in Fig. 7(b)), and the signal amplifier is used to collect polishing force and polishing temperature signals (as in Fig. 7(c)).

Table 1 Chemical composition of TC17 titanium alloy

\begin{tabular}{cc|cc}
\hline Ingredients & Contents (\%) & Ingredients & Contents $(\%)$ \\
\hline $\mathrm{Al}$ & $4.5-5.5$ & $\mathrm{Mo}$ & $3.5-4.5$ \\
$\mathrm{Sn}$ & $1.6-2.4$ & $\mathrm{Cr}$ & $3.5-4.5$ \\
$\mathrm{Zr}$ & $1.6-2.4$ & $\mathrm{Ti}$ & Bal. \\
\hline
\end{tabular}

\subsection{Experimental analysis}

Polishing force, polishing temperature and specific polishing energy are closely related to material machinability, material removal efficiency and surface quality. The influence of parameters on polishing force and polishing temperature is the basis for improving processing efficiency and surface quality.

The single factor method is used to study the influence of polishing speed $V_{s}$, compression quantity $a_{p}$, feed speed $f$, and size $p$ on polishing force $F$, polishing temperature $T$, specific polishing energy $e_{s}$ and surface roughness $R a$.

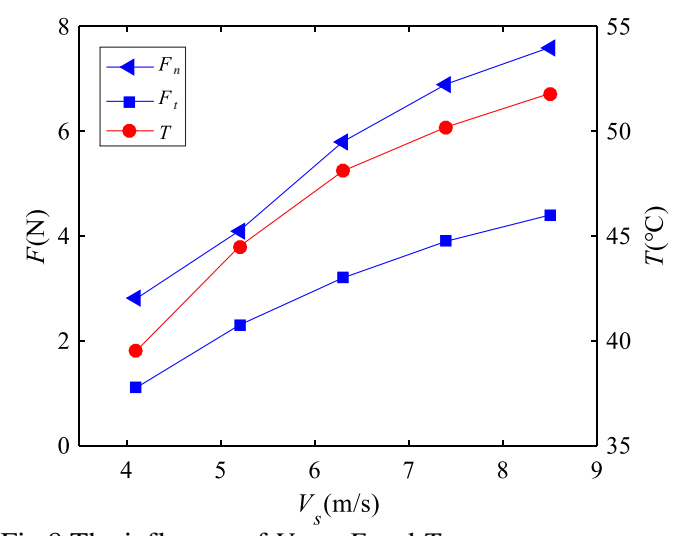

Fig.8 The influence of $V s$ on $F$ and $T$

As shown in Fig. 8, as the polishing speed increases, both the radial polishing force and the tangential polishing force show an increasing trend, and the radial polishing force changes more sensitively. The high-speed rotating flap disc obtains good flexibility under the action of centrifugal force and produces radial polishing force on the blade. As the polishing speed increases, the spindle speed has a greater rotation speed, and the flap disc obtains greater centrifugal force, resulting in a greater radial polishing force. The force of the flap disc on the blade is small, and the cutting depth of the abrasive particles is small. Therefore, the tangential resistance encountered during the rotation of the flap disc is small and the sensitivity of the tangential force to polishing speed is not obvious. As the polishing speed increases, the polishing temperature rises accordingly. The higher the surface polishing speed, the higher the limit stress of the material, and the higher the yield limit of the material. It makes material removal require more energy and generates more heat. In addition, as the polishing speed increases, the number of abrasive particles scratching on the blade surface per unit time will increase, which will also lead to an increase in polishing temperature.

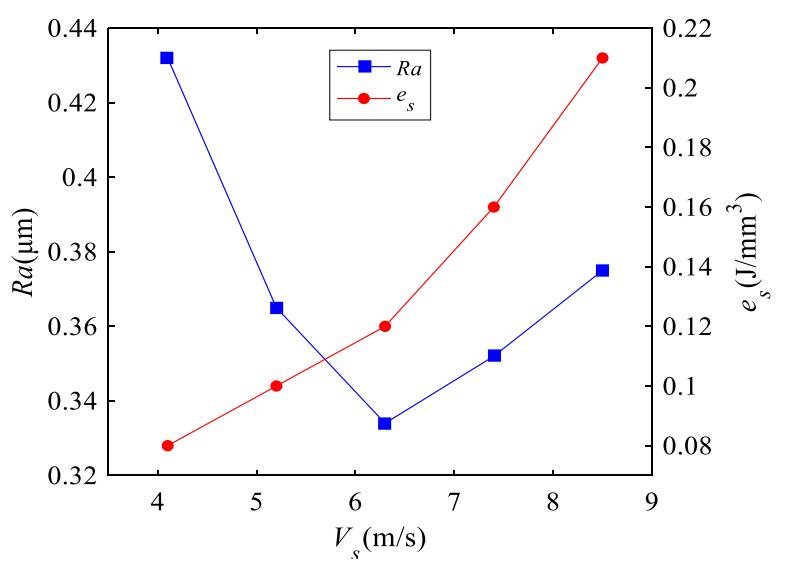

Fig.9 The influence of $V_{s}$ on $R a$ and $e_{s}$

As shown in Fig. 9, the roughness decreases at first and then increases with the increasing of polishing speed. When the polishing speed is small, the abrasive cloth piece on the flap disc is not fully expanded, and the flexibility of the flap disc is poor, resulting in a larger roughness. As the polishing speed increases, the flap disc obtains good flexibility and the polished surface roughness is reduced. As the polishing speed continues to increase, the polishing force of the flap disc is too large, which leads to the excessive material removal depth and the roughness becomes larger. The specific polishing energy increases with the increase of polishing speed. The specific polishing energy increases with the increase of polishing speed. The reason is that with the increase of polishing speed, the contact time between the blade and the abrasive cloth piece becomes shorter. The energy consumption of material removal per unit volume increases with the cutting form of abrasive grains change to ploughing form.

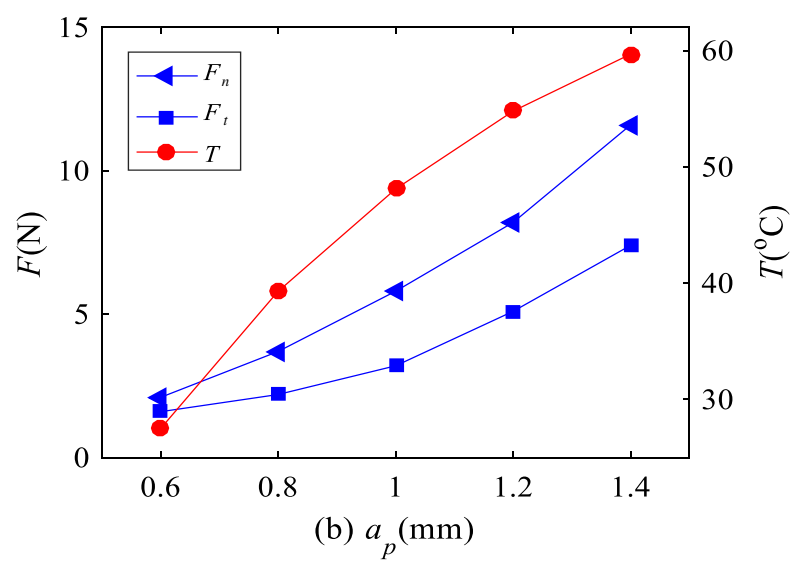

Fig.10 The influence of $a_{p}$ on $F$ and $T$

As shown in Fig. 10, as the compression quantity increases, both the axial polishing force and the radial polishing force show an upward trend. Analyzing the reasons for this change, the increase of compression quantity leads to an increase in 
the elastic deformation of the flap disc and the number of abrasive particles involved in polishing increases, which causes an increase in the radial polishing force. The increase of compression quantity leads to the increase of abrasive cutting depth, and more abrasive particles is removed into the cutting stage, resulting in a greater tangential polishing force. The increase of the tangential polishing force indicates increases of the friction between the flap disc and the blade, which causes the increase of polishing heat. Therefore, as the increases of compression quantity, the polishing temperature is improved.

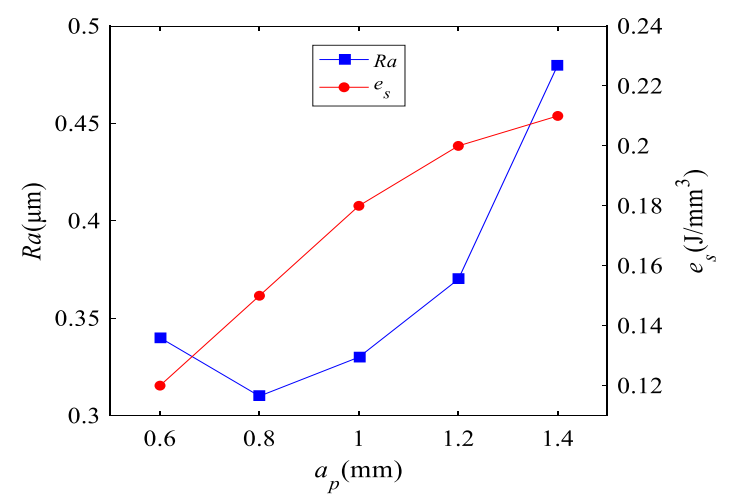

Fig.11 The influence of $a_{p}$ on $R a$ and $e_{s}$

As is in Fig.11, the roughness first decreases and then increases with the increase of compression quantity. The main reason is that when the compression quantity is small, the abrasive grains are mainly in the scratching stage, the amount of material removal is insufficient, the milling residual height cannot be completely removed, and the roughness is relatively large. As the compression quantity increases, the specific polishing energy is improved. The main reason may be the increase of the compression quantity, which accelerates the wear of abrasive grains, causes passivation of abrasive grains, and increases the energy consumption of removing materials per unit volume.

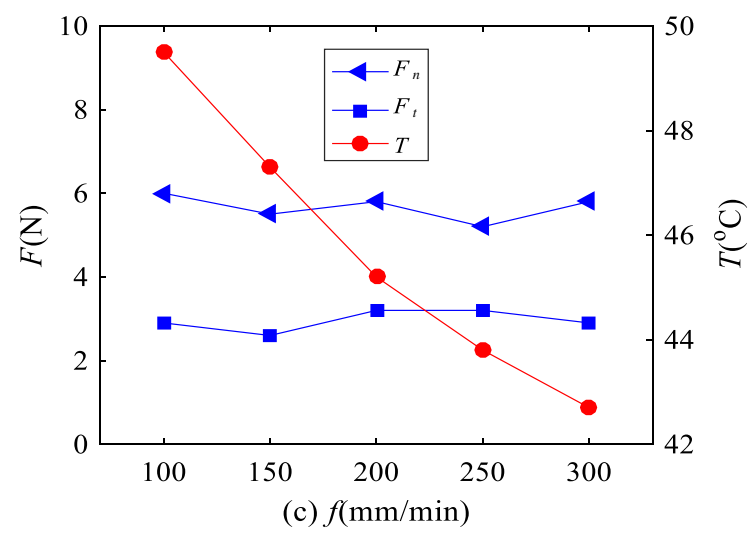

Fig.12 The influence of $f$ on $F$ and $T$

As is in Fig. 12, the increase of feed speed has no significant effect on polishing force. The reason is that the change of the feed speed has no effect on the elastic deformation of the flap disc and the material removal depth of the abrasive particles. Therefore, there is no significant change in the radial polishing force and the tangential polishing force. The polishing temperature decreases as the increase of feed speed. The reason is that as the feed speed increases, the heat dissipation conditions are improved. However, the friction between the blade and the flap disc does not change significantly, and the heat generated per unit time does not change significantly, so the polishing temperature is reduced.

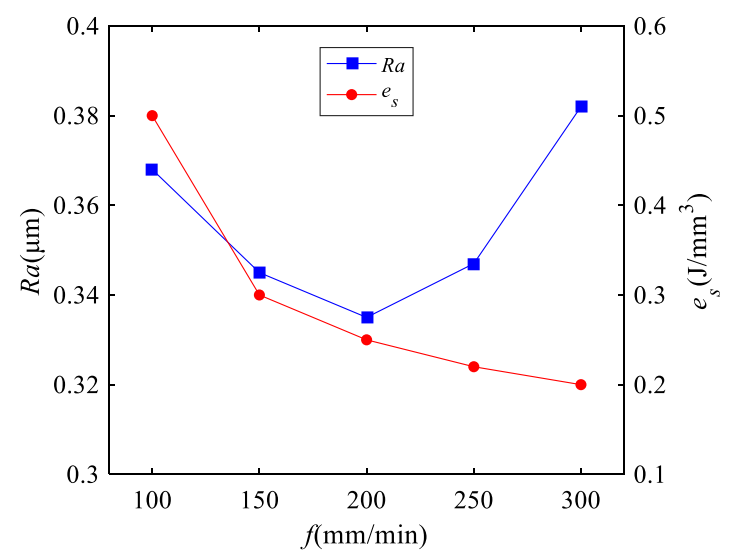

Fig. 13 The influence of $f$ on $R a$ and $e s$

As is in Fig. 13, the roughness first decreases and then increases with the increase of feed speed. The reason may be that when the feed speed is small, abrasive particles cut more times on the blade, and the material removal depth is greater, and excessive cutting depth is not conducive to forming a good polished surface quality. As the feed speed increases, the depth of material removal tends to be reasonable and the roughness is reduced. When the feed speed is too big, the cutting times of abrasive grains on the blade is too small, and it is insufficient to remove the milling residual height, resulting in a greater roughness. As the feed speed increases, the specific polishing energy is reduced. The main reason may be that as the increase of the feed speed abrasive particles are effectively contact with the milling residual height layer on the blade surface, the material removal efficiency of abrasive particles is improved, the energy of removing materials per unit volume is reduced, and the specific polishing energy is reduced. 


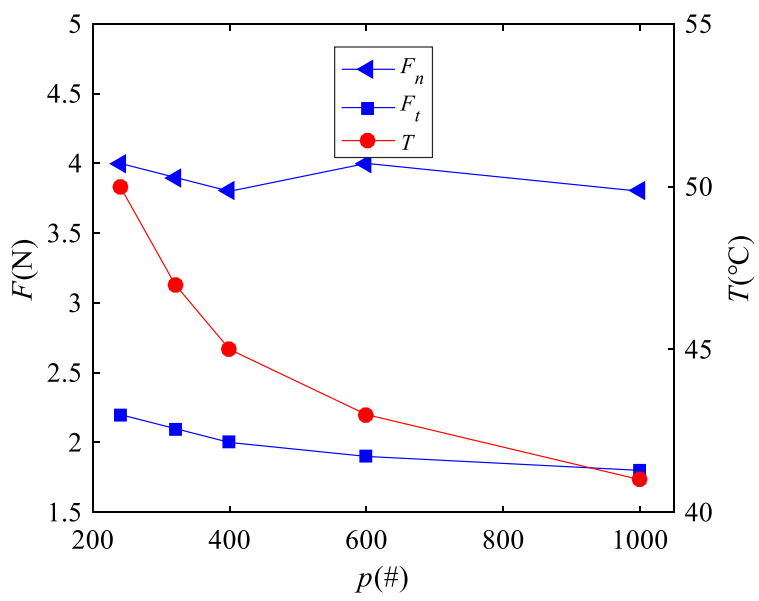

Fig. 14 The influence of $p$ on $F$ and $T$

The influence of size on polishing force and polishing temperature is shown in Fig. 14, with the increase of size, the radial polishing force does not change significantly, and the tangential polishing force increases slightly. The reason may be that as the size increases, the number of abrasive particles per unit area on the surface of the flap disc decreases, while the number of abrasive particles increases. Therefore, the change of size has little effect on the quality of the flap disc. When the spindle speed and compression quantity are constant, the radial polishing force does not change significantly. With the increase of the size, the material removal ability of the abrasive particles is weakened, and more abrasive particles are transformed into the scratch stage. The friction coefficient between the flap disc and the blade is reduced, so the tangential polishing force decreases. The polishing temperature decreases as the size increases, the reason may be that the radial friction of the flap disc to the blade does not change much and the friction coefficient is reduced. Therefore, the heat generated by the polishing is reduced, and the polishing temperature is reduced.

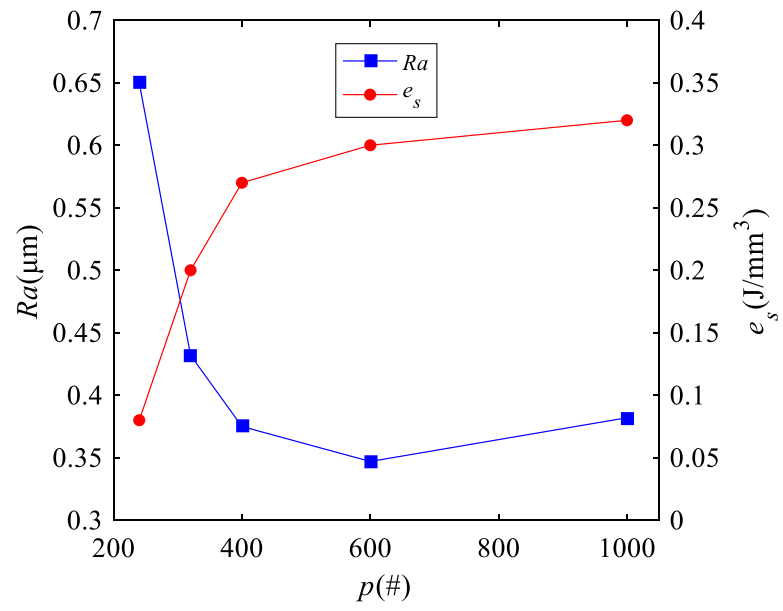

Fig. 15 The influence of $p$ on $R a$ and $e s$
The influence of size on roughness and specific polishing energy is shown in Fig. 15, the roughness first decreases and then increases with the increase of size. When the size is small, the volume of abrasive particles is larger, the material removal ability is stronger, the texture produced by polishing is more obvious, and the roughness is larger. As the particle size increases, the volume of abrasive particles decreases, the width and depth of material removal tends to be reasonable, the roughness decreases. As the size continues to increase, the amount of material removed by abrasive particles is not enough to remove the milling residual height of the blade, resulting in an increase in roughness. As the size increases, the specific polishing energy is improved. The main reason may be that as the increase of the size, the volume of abrasive particles is reduced, the material removal ability is reduced, and more energy is consumed to remove a unit volume of material.

\section{Polishing test verification}

According to the influence of process parameters on roughness, when the polishing speed is $6.2 \mathrm{~m} / \mathrm{s}$, the compression quantity is $0.8 \mathrm{~mm}$, the feed speed is $200 \mathrm{~mm} / \mathrm{min}$, and the size is 600 \#, the polished surface roughness is small. The optimized parameters are used for polishing experiments. The comparison of polishing test results are shown in Table 2. It can be seen from Table 2 that after parameter optimization, the polishing force is smaller, the polishing temperature is lower, and the roughness of the polished surface is smaller.

Table 2 Comparison of polishing test results

\begin{tabular}{cccccc}
\hline Category & $F_{n}$ & $F_{t}$ & $T$ & $e_{s}$ & $R a$ \\
\hline Traditional & 5.7 & 3.5 & 65 & 0.17 & 0.429 \\
Optimized & 4.2 & 2.5 & 45 & 0.24 & 0.372 \\
\hline
\end{tabular}

The comparison of traditional and optimized polished surface effect is shown in Fig. 16. The residual height of the milled blade surface is relatively large. The roughness of milled blade is $1.465 \mu \mathrm{m}$, and there are microscopic cracks on the surface. The width and thickness of traditional polishing debris are relatively large, the surface roughness of polished blade is $0.429 \mu \mathrm{m}$, and the texture is relatively obvious. After parameter optimization, the wear debris is relatively thin and small, the surface roughness is $0.372 \mu \mathrm{m}$, and the surface consistency is better.

The above analysis shows that after parameter optimization, a smaller polishing force is beneficial to reduce the size of wear debris and eliminate the surface texture of the blade. Besides, after optimization, the polishing temperature is lower, which is beneficial to reduce the thermal deformation of the workpiece surface, and the processing accuracy is improved. Therefore, after optimization, the polished surface 
has a smaller roughness and a higher consistency.

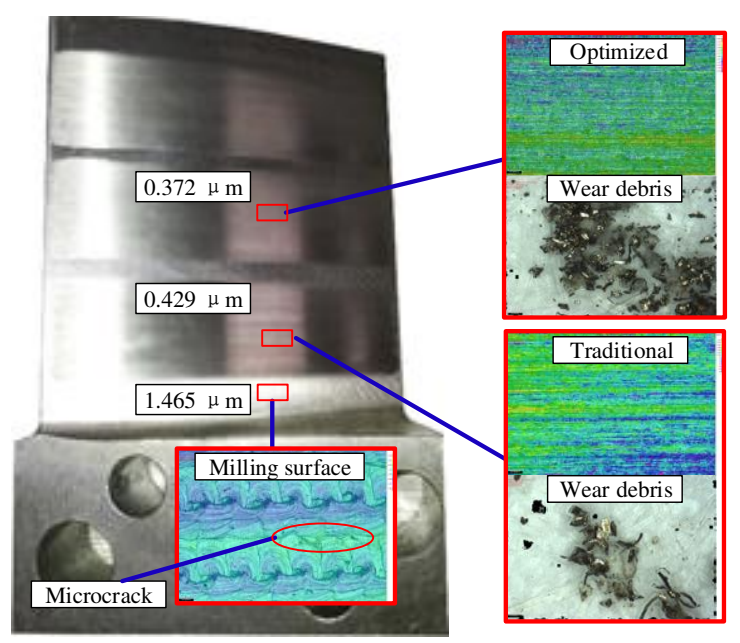

Fig. 16 The comparison of traditional and optimized polished surface

\section{Conclusions}

The principle of flexible polishing of the elastic spindle is analyzed, the cutting process model of the flap disc and the model of the influence of the flap disc elastic deformation on the contact arc length are established.

The analytical model for the polishing force and polishing temperature of the flap disc is established. The experiment shows that as the polishing speed increases, the polishing force, polishing temperature and specific polishing energy increase, and the roughness first decreases and then increases. As compression quantity increases, the polishing force, polishing temperature and the specific polishing energy increase, and the roughness first decreases and then increases. As the feed speed increases, the polishing force does not change significantly, the polishing temperature and specific polishing energy decrease, and the roughness first decreases and then increases. With the increase of size, the radial polishing force does not change much, the tangential polishing force and temperature decrease, the specific polishing energy increases, and the roughness first decreases and then increases.

The traditional and the optimization polishing experiments are compared, after optimization, the polishing force, polishing temperature and polishing surface roughness are improved, but the specific polishing energy is increased. Besides, after the optimization, the size of the wear debris is smaller and the consistency is higher.

\section{Declarations}

Funding This research was supported by the Zhejiang
Provincial Natural Science Foundation of China under Grant No. LY20E050027 and the Zhejiang Provincial Natural Science Foundation of China under Grant No. LY19F030006.

Conflicts of interest/Competing interests I would like to declare on behalf of my coauthors that the work describes original research that has not been published previously and is not under consideration for publication elsewhere, in whole or in part.

Availability of data and material The material selection and data obtained in this manuscript have been tested or calculated theoretically, which are true and reliable.

\section{Code availability Not applicable.}

Authors' contributions The abrasive cutting model of the flap disc is established. The theoretical calculation of the effect of elastic deformation during the polishing process on the contact length. The model of polishing force, polishing heat and temperature field during the polishing process of the flap disc are established and analyzed. Single factor method is used to analyze the influence of process parameters on polishing force, polishing temperature, roughness and specific polishing energy.

Ethics approval The submission of this manuscript involves no conflicts of interest, and the manuscript has been approved for publication by all authors.

Consent to participate All the authors listed have approved the manuscript as enclosed.

Consent for publication We would like to submit the enclosed manuscript, entitled "Study on flexible polishing force and polishing temperature of flap disc", which we wish to be considered for publication in The International Journal of Advanced Manufacturing Technology.

\section{References}

1. Wu XJ, Tong X, Sun H (2018) Parameter Optimization of Polishing M300 Mold Steel with an Elastic Abrasive. Math Pro Engi, 2018:1-9

2. Xian, C, Shi, Y, Lin X and Liu D (2020) Study on vibration characteristics polishing rod for polishing aeroengine blade with abrasive cloth wheel. Math Prob Engin 2020(90): 1-14 
3. Lin X, Yang R, Xin X (2020) Modeling and Parameter Optimization of Flexible NC Polishing Vibration of Abrasive Cloth Wheel Based on Sensitivity Analysis. Math Prob Engi 2020(6):1-12

4. Wang C, Guo H, Zhao Y, Sun Q, Zhao L (2018) Statistical analysis of industrial grinding brush force characteristics based on finite element approach. Math Prob Engin, 2018(3):1-9

5. Liu D, Shi Y, Lin X, Xian C (2019) Study on improving surface residual stress of polished blade after polishing based on two-stage parameter method. The International Journal of Adv Manu Tech, 100(5):14911503

6. Liu D, Shi Y, Lin X, Xian C, Gu Z (2020) Polishing surface integrity of TC17 aeroengine blades. J Mech Sci Tech, 34(2): 689-699

7. Liu D, Shi Y, Lin X, Xian C, Gu Z (2020) Optimization Analysis of Blade Polished Surface Roughness and Residual Stress. Com Int Manu Sys, 27(5):13281340

8. Zhang J, Shi Y, Lin X, Li Z (2017) Parameter optimization of five-axis polishing using abrasive belt flap wheel for blisk blade. J Mec Sci Tech, 31(10):48054812

9. Liu D, Shi Y, Lin X, Xian C, Gu Z (2019) Study on avoiding the knife marks of the blade after polishing by flap disc. Int J Adv Man Tech, 105(5-6):27332744

10. Wang Z, Lin X, Shi Y, Zhang Y, Chen Z (2020) Reducing roughness of freeform surface through tool orientation optimization in multi-axis polishing of blisk. Int J Adv Man Tech, 108(1-4):1-13

11. Huai W, Tang H, Shi Y, Lin X (2017) Prediction of surface roughness ratio of polishing blade of abrasive cloth wheel and optimization of processing parameters. Int Journal Adv Man Tech, 90(1-4):699-708

12. Huai W, Shi Y, Tang H, Lin X (2017) Sensitivity of surface roughness to flexible polishing parameters of abrasive cloth wheel and their optimal intervals. $\mathrm{J}$ Mech Sci Tech, 31(2):865-873

13. Ma CP. Guan YC, Zhou W (2017) Laser polishing of additive manufactured Ti alloys. Opti Lase Engi, 93:171-177

14. Xiao G, Huang Y (2015) Constant-load adaptive belt polishing of the weak-rigidity blisk blade. Int J Adv Man Tec, 78(9-12):1473-1484

15. Tian F, Li Z, Lv C, Liu G (2016) Polishing pressure investigations of robot automatic polishing on curved surfaces. Int J Adv Man Tech, 87(1-4):639-646

16. Janus J, Fauxpoint G, Arntz Y (2010) Surface roughness and morphology of three nanocomposites after two different polishing treatments by a multi technique approach. Den Mat, 26(5):416-425

17. Antonson SA, Yazici AR, Kilinc E (2011) Compari- son of different finishing/polishing systems on surface roughness and gloss of resin composites. J Den, 39: e9-e17

18. Gönülol N, Y1lmaz F (2012) The effects of finishing and polishing techniques on surface roughness and color stability of nanocomposites. J dent, 40: e64-e70

19. Bordatchev EV, Hafiz AMK (2014) Tutunea-Fatan OR Performance of laser polishing in finishing of metallic surfaces. Int J Adv Manu Tech, 73(1-4):3552

20. Berger SB, Palialol A, Cavalli V, Giannini M (2011) Surface roughness and staining susceptibility of composite resins after finishing and polishing. J Est Rest Den, 23(1):34-43

21. Zhang J, Shi Y, Lin X (2017) Five-axis abrasive belt flap wheel polishing method for leading and trailing edges of aero-engine blade. Int J Adv Manu Tech, 93(9-12): 3383-3393

22. Huai W, Lin X, Shi Y (2020) Geometric characteristic modeling for flexible contact of sanding wheel-polished complex surface. Int J Adv Man Tec, 2020, 110(2):1691-1700 